PSICOLOGIA

IBEROAMERICANA
Psicología Iberoamericana ISSN: 1405-0943

revista.psicologia@ibero.mx

Universidad Iberoamericana, Ciudad de México México

\title{
Aproximación ecológica a la negligencia infantil en Puerto Rico: una identificación de riesgos desde la perspectiva profesional
}

Mulero Ortiz, Alexandra; Montero-López Lena, María

Aproximación ecológica a la negligencia infantil en Puerto Rico: una identificación de riesgos desde la perspectiva profesional

Psicología Iberoamericana, vol. 28, núm. 1, 2020

Universidad Iberoamericana, Ciudad de México, México

Disponible en: http://www.redalyc.org/articulo.oa?id=133963198004 
Artículos

\title{
Aproximación ecológica a la negligencia infantil en Puerto Rico: una identificación de riesgos desde la perspectiva profesional
}

Ecological approach to child neglect in Puerto Rico: A risk identification from a professional perspective

\author{
Alexandra Mulero Ortiz mulero.alexandra@gmail.com \\ Universidad Nacional Autónoma de México (UNAM), México, México \\ (D) http://orcid.org/0000-0002-3204-8709 \\ María Montero-López Lena \\ Universidad Nacional Autónoma de México (unam), México, México \\ http://orcid.org/0000-0001-9109-381X
}

Psicología Iberoamericana, vol. 28, núm. 1,2020

Universidad Iberoamericana, Ciudad de México, México

Recepción: 05 Noviembre 2019 Aprobación: 24 Abril 2020

Redalyc: http://www.redalyc.org/ articulo.oa?id $=133963198004$
Resumen: El estudio tuvo por objetivo identificar factores que promueven la manifestación de negligencia infantil desde la perspectiva profesional y proponer un modelo conceptual para futuras apoximaciones a esta modalidad de maltrato. Se analizan modelos conceptuales que abordan la etiología del maltrato hacia niños(as) y se explora la perspectiva profesional sobre factores de riesgo asociados con la negligencia. La metodología seguida fue de corte cualitativo, empleando la técnica de entrevista electrónica estructurada y de análisis temático. Participaron en el estudio 52 profesionales que laboran en instituciones dedicadas al cuidado de niños en Puerto Rico. Los resultados indican que variables en diferentes niveles, desde la salud mental de los padres hasta la inequidad social, pueden promover eventos de negligencia infantil. La detección de variables críticas en dichos niveles contribuye a proponer un abordaje ecológico para el estudio de la negligencia infantil.

Palabras clave: negligencia infantil, teoría ecológica, entrevista estructurada, salud mental parental, factores de riesgo.

Abstract: This study aimed to identify risk factors that foster child neglect and to suggest a conceptual framework for future studies on this type of child maltreatment. Conceptual models on the etiology of child neglect are explored as well as the professional perspective on risk factors associated with child neglect. A total of 52 professionals that worked on institutions related to childcare in Puerto Rico answered a structured electronic interview on what they consider to be causes of child neglect. A thematic analysis was conducted, and results show that variables on different levels, from caretaker's mental health to social inequality, may promote child neglect. Findings contribute to the proposal of an ecological approach to identify critical variables in specific interaction levels that work as catalysts for neglectful events.

Keywords: child neglect, ecological theory, structured interview, parental mental health, risk factors.

\section{Introducción}

El Fondo de las Naciones Unidas para la Infancia define la negligencia infantil como un tipo de maltrato que, en contraste con el abuso, "es el 
resultado de la falta de atención a las necesidades básicas de un niño, aun cuando se cuenta con los medios, el conocimiento y el acceso a servicios para atenderlas" (UNICEF, 2014, p. 4). La negligencia se ha descrito como un fenómeno heterogéneo que varía en cuanto a tipo, severidad y cronicidad (Dubowitz, Black, Starr, \& Zuravin, 1993). Algunos tipos de negligencia son la negligencia física, la emocional, médica, educativa, nutricional, psicológica y ambiental (Sciarrino, Hernández, \& Davidtz, 2018, p. 3).

En Puerto Rico, la negligencia representa el mayor porcentaje de los casos de maltrato fundamentados (Disdier, Lugo, \& Irizarry, 2015), alcanzando según los datos más recientes, el 62\% en el año 2015 (U.S. Department of Health \& Human Services, Administration for Children and Families, Administration on Children, Youth and Families [ACF], $\&$ Children's Bureau, 2016).

Estudios recientes sobre el maltrato infantil en Puerto Rico se han enfocado en la descripción de sus características (Ishida, Klevens, Rivera-García, \& Mirabal, 2013), el manejo de referidos de maltrato (Concepción Lizardi \& Sánchez Peraza, 2017), intervenciones dirigidas a reducir el riesgo de maltrato infantil (Sánchez Cesáreo, Sánchez Cardona, Beyer, González Jiménez, \& Bensinger, 2018; Sánchez Cesáreo et al., 2016), la relación entre el maltrato infantil y la depresión en jóvenes (Jaschek, Carter-Pokras, He, Lee, \& Canino, 2016), tasas de prevalencia de maltrato y predictores sociodemográficos y culturales de maltrato infantil (Sledjeski, Dierker, Bird, \& Canino, 2009). En dos de estas publicaciones se identifican posibles factores de riesgo para el maltrato infantil. Entre estos, se mencionan la pobreza (Sledjeski et al., 2009), el desempleo y la estructura familiar uniparental (Sánchez Cesáreo et al., 2018). No obstante, la literatura sobre factores de riesgo para la negligencia, en particular, en el contexto puertorriqueño, es escasa.

En la literatura internacional se ha documentado la presencia de factores de riesgo para la negligencia a nivel familiar, comunitario y social (Sciarrino et al., 2018, p. 7). En los Estados Unidos, la negligencia suele asociarse con variables que evidencian pobreza o dificultades económicas reales (Slack et al., 2011) y percibidas (Barnhart \& Maguire-Jack, 2016), tales como el desempleo, la falta de apoyo económico institucional (Child Welfare Information Gateway, 2012), la falta de hogar o de una residencia apropiada, entre otras. Esta relación es de especial interés para el caso de Puerto Rico, dados los niveles de desigualdad y pobreza que enfrenta su población.

Actualmente en Puerto Rico, el $46 \%$ de las familias y el $43 \%$ de las personas viven bajo el nivel de pobreza (Instituto de Estadísticas de Puerto Rico, 2016). De acuerdo con el censo poblacional más reciente, de un total de 841,740 personas menores de 18 años, 56\% vivía en pobreza (Children's Bureau, 2013). A este contexto se le añade lo que Belsky (1993, p. 423) considera como circunstancias que devalúan la labor de velar por el bienestar de los niños y que pueden exceder las capacidades de muchos cuidadores(as). Las condiciones mencionadas pueden resumirse en el cierre injustificado de más de 300 escuelas (Associated Press, 2017), 
el incumplimiento con la asignación de psicólogos escolares (El Vocero, 2017), la precariedad laboral a la que se exponen las maestras y el estado de abandono en el que se mantienen los hogares de crianza (Ávila-Claudio, 2017).

Estos y otros problemas se agravaron luego del paso de dos huracanes en el año 2017. La secuela de los huracanes implicó nuevas adversidades, como pérdidas de seres queridos, redes sociales y daños materiales extensos para un gran número de familias. Lo anterior, aunado a la falta de servicios sociales, puede afectar la atención de las necesidades de los niños y las capacidades de crianza de los adultos (Kilmer \& Gil-Rivas, 2010, p. 139).

En Puerto Rico, ante la alta cifra de negligencia infantil y la falta de literatura referente a los factores de riesgo para este fenómeno, es necesario el desarrollo de modelos conceptuales que permitan una mejor comprensión de sus causas y consecuencias. La presente investigación analiza algunos de los modelos conceptuales que se han propuesto para explicar el abuso y la negligencia; además, explora la perspectiva profesional sobre factores de riesgo asociados a esta.

\section{Abordajes teóricos}

El abuso y la negligencia se han abordado desde, al menos, cuatro dominios conceptuales que dan lugar a cuatro tipos de modelos: psiquiátricos, sociológicos, interaccionales y ecológicos. En este apartado se hará énfasis en la negligencia, pero es importante tener presente que los modelos expuestos a continuación suelen contemplar el abuso y la negligencia como una misma variable dependiente.

\section{Modelos psiquiátricos o psicológicos}

Estos modelos fueron frecuentes en los primeros estudios sobre la etiología de la negligencia y se centraron sobre todo en características parentales o familiares. Se enfocan en las características psicológicas de los padres maltratantes, contemplando patologías, trastornos mentales y de personalidad. Torío-López y Peña-Calvo (2006) resaltan que, en cierta forma, se responsabilizaba a la biología de lo sucedido, adjudicando el comportamiento maltratante a una falta de recursos para desempeñar el rol de padre o madre.

Los modelos de deficiencias parentales hacen énfasis en algunos comportamientos inadecuados de crianza, y señalan que las interacciones inapropiadas entre el menor y su cuidador son producto de características parentales o familiares particulares (Smith \& Fong, 2004). Spinetta y Rigler (1972) fortalecieron esta postura basándose en una revisión que reveló que (a) los cuidadores/perpetradores habían sido criados bajo algún grado de privación; (b) traían a su rol de padres nociones equívocas de crianza; (c) tenían un defecto en la estructura de carácter que permitía que sus impulsos agresivos se manifestaran libremente; y (d) aunque los factores socioeconómicos pudieran añadir estrés sobre las debilidades en 
sus personalidades, no eran causas en sí mismas, suficientes o necesarias del abuso.

De estos modelos se derivan las propuestas de maltrato intergeneracional, en donde se plantea una relación entre la victimización pasada de los cuidadores y la perpetración de maltrato hacia sus hijos. Estudios como los de Oliveira, Maroco y Pais (2012) y el de Thornberry y Henry (2013) evidencian esta asociación. Es decir, personas que habían sido maltratadas en algún momento en su niñez o adolescencia, tenían una mayor probabilidad de maltratar a sus hijos que aquellos que no habían sido maltratados. En el estudio de Thornberry y Henry (2013) esta relación era más fuerte en los casos de maltrato que ocurrieron en la adolescencia o que comenzaron en la niñez y persistieron durante la adolescencia. Por su parte, Kim (2009) documentó que los padres que reportaban haber sido víctimas de negligencia en su niñez, contrario a los que no habían tenido esa experiencia, tenían una mayor probabilidad de describirse como negligentes con sus hijos y de reportar estilos de crianza abusivos.

La teoría psicopatológica ha sido foco de críticas por diversas razones, entre ellas, porque ofrece una explicación muy estrecha del maltrato, ignorando otras variables que pueden ser igual o más importantes para explicarlo. Al respecto, Gelles (1973) criticó la teoría psicopatológica del abuso infantil, ya que consideró que plantear una variable independiente única, como una enfermedad mental, ignoraba otras variables que eran de igual o mayor importancia para comprender el fenómeno. Además, Gelles consideró inconsistente a la teoría psicopatológica, dado que la mayoría de los perpetradores identificados no eran psicópatas. Como respuesta a estas consideraciones, surgieron otros modelos que buscan las causas del maltrato fuera del individuo.

\section{Modelos sociológicos}

Los modelos sociológicos analizan las condiciones de maltrato, desde el contexto sociocultural en el que ocurre (Wiehe, 1989), y parten de la premisa de que son las deficiencias en las estructuras sociales donde habitan las familias las que generan condiciones de maltrato (Baumrind, 1994). Gelles (1973) propuso un modelo en el que el maltrato surge como una respuesta al estrés provocado por la relación de pareja de los padres, característica estructural y particularidad del menor.

Por otra parte, para Gil (1969) el maltrato infantil es un fenómeno que se concentra principalmente en los sectores más vulnerables de la sociedad. Martin y Walters (1982) evidenciaron una relación positiva entre la negligencia infantil y circunstancias intrafamiliares caracterizadas por la pobreza. Al combinar variables que indican un bajo nivel socioeconómico, como problemas financieros temporales y una cantidad numerosa de hijos para el espacio disponible en el hogar o para el monto de ingreso familiar, se explica una cantidad moderada de la varianza total. Más aún, enfatizan que estos factores parecen predecir la negligencia infantil más que otros tipos de maltrato. 
Otros estudios recientes han confirmado la relación entre el maltrato y la situación económica de las familias. Por ejemplo, aumentos exógenos en el ingreso familiar pueden asociarse con disminuciones modestas en los comportamientos negligentes y con una reducción significativa en intervenciones de agencias de protección infantil (Berger, Font, Slack, $\&$ Waldfogel, 2016). Otros estudios han concluido que la desigualdad económica se asocia de manera significativa con altos niveles de maltrato infantil (Eckenrode, Smith, McCarthy, \& Dineen, 2014).

Los modelos sociológicos o de deficiencias ambientales han sido muy útiles para profundizar en factores relacionados con la negligencia fuera del sistema familiar. No obstante, sugiere Belsky (1978) que familias expuestas a estresores sociales similares a los mencionados, no recurren al maltrato. Dicho autor insiste en que, pese a lo adecuado que parecería ser el modelo sociológico para explicarlo, no aclara por sí mismo el maltrato. Es importante que se considere entonces el rol que juegan las características del niño en los eventos de maltrato y negligencia.

\section{Modelos interaccionales}

Los modelos interaccionales asumen una relación bidireccional en la que padres e hijos son participantes activos en el proceso de maltrato o negligencia (Wiehe, 1989; Pardeck, 1989). Por lo mismo, además de contemplar las características de los cuidadores, estos modelos integran características de los niños que les exponen a un mayor riesgo de maltrato. Los modelos interaccionales se asocian con la teoría de coerción de Patterson (1982), la cual describe un proceso mutuo en el que los cuidadores refuerzan los comportamientos "difíciles" de los niños, lo que evoca una reacción negativa del cuidador, hasta que la interacción se detiene cuando uno de los participantes "gana" (Smith et al., 2014). Según indican estos autores, este ciclo puede comenzar cuando el niño reacciona con molestia o resistencia a una instrucción o petición del cuidador, lo que provoca enojo u hostilidad en el cuidador, reacciones que usualmente se intensifican a medida que el ciclo de coerción va escalando.

Para Fattah (1989, p. 200) hay dos aspectos claves en la interacción cuidador-hijo que podrían conllevar a una situación de maltrato: 1) la falta de habilidades para manejar efectivamente situaciones cotidianas de crianza o disciplina y 2) una alta proporción de comportamientos hostiles por parte del cuidador y del hijo. En el caso de la negligencia, sería posible argumentarse que la misma falta de habilidades del cuidador podría generar, ya no una reacción hostil, sino una convicción de que el hijo no requiere ciertos cuidados.

Por otra parte, ciertas características de los niños los hacen más vulnerables al maltrato. Friedrich y Boriskin (1976) mencionan, por ejemplo, a niños prematuros, con retraso mental, discapacidades físicas y comportamientos o temperamentos atribuidos a contribuciones genéticas. Indican que dichas condiciones están sobre representadas en las poblaciones de niños maltratados. Además, la percepción que tiene el padre/madre de su hijo(a) como "diferente" de sus hermanos(as) o pares, 
ya sea por una de las características mencionadas, o porque no es su hijo(a) biológico(a), lo coloca en una posición de mayor vulnerabilidad, pues el cuidador puede verse incapacitado para manejar o interactuar con su hijo(a).

Los modelos interaccionales, al igual que los psiquiátricos y sociológicos, abordan la etiología de la negligencia desde una perspectiva única. Además, gran parte de la investigación sobre maltrato y negligencia suele fundamentarse o utilizar muestras de familias que ya están involucradas con agencias de servicios de protección para niños. Esto podría estar provocando un sesgo de muestreo que obstaculiza la identificación de otras variables críticas (Platt, 1964) que podrían incidir sobre la desatención de las necesidades de los niños.

$\mathrm{Si}$ bien los tres modelos descritos han aportado en gran medida a la identificación de variables críticas, autores como Belsky (1980) han propuesto que el maltrato infantil está determinado por múltiples factores que afectan al individuo, la familia, la comunidad y la cultura. Wiehe (1989) sugiere que el abordaje ecológico puede fungir como un modelo integrador, sin perder de perspectiva la totalidad del problema.

\section{Modelos ecológicos o transaccionales}

Los modelos ecológicos o transaccionales ubican la negligencia en un contexto ecológico conformado por múltiples niveles de análisis. Esos niveles incluyen el macrosistema, el exosistema, el microsistema y el nivel de desarrollo ontogénico (Figura 1). Entre estos, suelen incluirse características individuales de cuidadores y niños, las interrelaciones cuidador-menor, la situación específica de vida, las instituciones sociales y la cultura del contexto donde se desenvuelven las familias. Como puede apreciarse, los niveles de análisis se encuentran anidados y con diferentes grados de proximidad al individuo (Bronfenbrenner, 1979; Belsky, 1980; Lynch \& Cicchetti, 1998). 


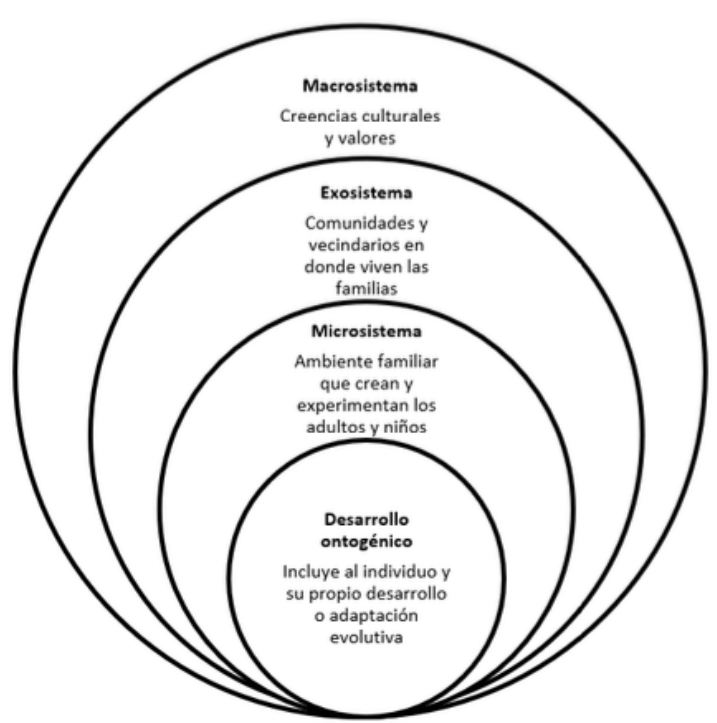

Figura 1. Representación esquemática del contexto ecológico del maltrato hacia niños (Lynch \& Cicchetti, 1998, p. 235).

Figura 1. Representación esquemática del contexto ecológico del maltrato hacia niños (Lynch \& Cicchetti, 1998, p. 235)

Se propone que cada nivel de análisis contiene factores de riesgo y factores protectores para el individuo. Para Lynch y Cicchetti (1989) los factores de riesgo pueden influir directamente sobre el individuo, pero también sobre eventos que suceden en los diferentes subsistemas. Los factores de riesgo aumentan la probabilidad de un desarrollo deficiente, mientras que los factores protectores disminuyen el riesgo de ese resultado (Lynch \& Cicchetti, 1989). Vondra y Toth (1989) explican que el balance acumulativo entre esos factores es lo que determina las diferencias individuales en la crianza. Por lo mismo, se entiende que el maltrato ocurre cuando los factores de riesgo sobrepasan a los protectores o compensatorios (National Research Council, 1993).

Una de las bondades de los modelos ecológicos es que buscan identificar las causas suficientes y necesarias para que ocurra el maltrato. Según Bronfenbrenner y Mahoney (1975), para que una condición suficiente provoque un efecto o consecuencia específica, todas las condiciones necesarias relevantes deben estar presentes. La ausencia de éstas "desarma" de forma efectiva a las condiciones suficientes. Tomando esto en cuenta, Garbarino (1977) concluye que las condiciones necesarias para que exista el maltrato infantil en microsistemas familiares son: 1) la justificación cultural para el uso de fuerza contra los niños y 2) el aislamiento de la familia de sistemas potenciales de apoyo, ya sea porque no están disponibles o porque fallan en hacer uso de ellos.

Por su parte, Lynch y Cicchetti (1998) proponen un modelo ecológico transaccional para analizar la relación entre el maltrato hacia niños, la violencia comunitaria y el funcionamiento de los niños. Mediante una prueba empírica concluyen que hay una influencia mutua entre los niños y sus contextos. Por ejemplo, encontraron que las incidencias de maltrato 
estaban relacionadas con los niveles de violencia comunitaria reportada por los niños. Así mismo, tanto el maltrato como la violencia comunitaria se asociaron con el funcionamiento de los niños.

Según Dubowitz (2009), los abordajes ecológicos reconocen que hay una multiplicidad de factores que interactúan entre sí y contribuyen a los estilos de crianza, así como a la negligencia. Muchos de los factores contribuyen a la negligencia y están fuera del alcance de los cuidadores, tales como fallas en los sistemas económicos, sociales, educativos y de salud (Proctor \& Dubowitz, 2014). Como se mencionó, los modelos que abordan la negligencia desde una perspectiva única han sido útiles para identificar una diversidad de variables críticas. Ante la ausencia de un sistema integrador de esos abordajes divergentes, Belsky (1980, p. 320) propone el modelo ecológico como uno capaz de ordenarlos y de abarcar la complejidad de los contextos de crianza de niños.

En su plan para la prevención del maltrato, el Departamento de la Familia de Puerto Rico, responsable de atender los casos de maltrato y negligencia, ha adoptado el modelo ecológico "como una herramienta para analizar la naturaleza de violencia" (Departamento de la Familia, 2014, p. 8). Con este modelo, buscan explicar las múltiples causas de la violencia y lo utilizan como marco de referencia para contextualizar las diferentes respuestas de prevención de maltrato. Sin embargo, reconociendo que la negligencia es un fenómeno que puede estar relacionado, pero no es igual al abuso físico, sexual y otras modalidades de maltrato, se debe recopilar información exclusiva y contextualizada de este fenómeno.

Los educadores, psicólogos, trabajadores sociales, entre otros profesionales responsables de velar por el bienestar de los niños, suelen ser quienes establecen y comunican a la población general, a través de diversos medios, lo que constituyen cuidados y comportamientos aceptables hacia los niños (Williams, 2017). Además, los maestros, directores y miembros de las comunidades escolares tienen la responsabilidad legal de intervenir y notificar a las autoridades pertinentes si hay señales de maltrato o negligencia hacia algún menor. Es decir, los profesionales deben tener una preparación que les permita identificar niños en riesgo de negligencia.

La opinión y experiencia profesional son esenciales para comprender el constructo de negligencia infantil, cómo se identifica y se maneja. En el caso de Puerto Rico, la Ley para la Seguridad, Bienestar y Protección de Menores establece que las agencias de gobierno, como el Departamento de Salud, Departamento de la Familia, Departamento de Educación, entre otros, deben coordinar esfuerzos entre sí "cuando se requiera la prestación de servicios relacionados a la identificación, prevención o tratamiento de los menores que son víctimas de maltrato, maltrato institucional, negligencia o negligencia institucional" (Ley No 246, 2011, p. 12). Es a los funcionarios públicos a quienes se les delega la identificación y manejo de casos de maltrato y negligencia. Por esta razón, el objetivo de este estudio fue identificar factores de riesgo para la negligencia hacia niños desde la perspectiva profesional. En específico, se adoptó un abordaje ecológico 
para identificar variables en distintos niveles que podrían potencializar la negligencia infantil.

El modelo ecológico provee un marco conceptual que busca dar cuenta de la complejidad inherente a los contextos de desarrollo de los niños (Lynch \& Cicchetti, 1998, p. 236). De acuerdo con Bronfrenbrenner (1979), el ambiente es concebido como un conjunto de sistemas anidados que interactúan entre sí y edifican la realidad social que vive el individuo en desarrollo. Ese ambiente está conformado por cuatro sistemas de interacción: microsistema, mesosistema, exosistema y macrosistema.

El microsistema se define como un patrón de interacciones recíprocas que experimenta un individuo en un entorno específico (Bronfrenbrenner, 1979, p. 41). En ese sistema se incluyen características individuales del cuidador y del niño, las cuales generan respuestas y modifican las conductas entre ambos. El mesosistema puede considerarse "un sistema de microsistemas" (Bronfrenbrenner, 1979, p. 44) y se caracteriza por las interrelaciones entre dos o más entornos en los que el individuo participa de forma activa. Ejemplos de este sistema podrían ser la interacción del cuidador con amistades del trabajo, o del niño con amistades del vecindario en el que viven.

Un exosistema se refiere a "uno o más entornos que no incluyen a la persona en desarrollo [...] pero en los cuales se producen hechos que afectan lo que ocurre en el entorno que comprende a la persona en desarrollo" (Bronfrenbrenner, 1979, p. 44). Un exosistema podría ser el lugar de trabajo de los padres. Por último, el macrosistema incluye los valores culturales y creencias que dan paso a la violencia intrafamiliar y en comunidades (Lynch \& Cicchetti 1998). Explica Bronfrenbrenner (1979, p. 28) que "la política oficial es una parte del macrosistema que determina las propiedades específicas del exo-, el meso- y el microsistema, que ocurren a nivel de la vida diaria y dirigen el curso de la conducta y el desarrollo".

\section{Método}

Este estudio cualitativo tuvo por objetivo conocer factores de riesgo para la negligencia infantil desde la experiencia laboral y perspectiva de profesionales puertorriqueños. El diseño del estudio fue exploratorio descriptivo, empleando un análisis temático interpretativo (Smith \& Osborn, 2003) de los datos recabados.

\section{Procedimiento e instrumento}

Se diseñó un cuestionario breve con el que se pudieran identificar modalidades de negligencia presentes en el contexto puertorriqueño, enumerarlas junto con factores de riesgo que promuevan la negligencia. El contenido fue validado por dos jueces expertos en la temática. Posteriormente el cuestionario se adaptó a un formato digital, en el portal web esurveycreator.com, y se difundió tanto por redes sociales como por 
correo electrónico a personas de interés, tales como trabajadores sociales, psicólogas(os), maestras(os), enfermeras(os) y otros profesionales cuya labor estuviera relacionada con la protección de niños en Puerto Rico.

El cuestionario estuvo disponible en línea entre los meses de agosto y noviembre del año 2018; incluía una página de presentación en la que se explicaba el propósito del estudio y se obtenía el consentimiento informado de los participantes. Los profesionales participaron de manera voluntaria y con conocimiento pleno de que toda la información ofrecida sería tratada de manera anónima y confidencial.

El cuestionario contenía una breve sección de datos demográficos y una definición de negligencia seguida por tres preguntas abiertas y una sección de comentarios:

1. ¿Qué situaciones de negligencia le ha tocado ver en su trabajo?

2. ¿Qué modalidades de negligencia son las más comunes en Puerto Rico?

3. ¿Cuáles diría son las principales causas de la negligencia hacia niños? En el cuestionario se definió la negligencia según propone el Fondo de las Naciones Unidas para la Infancia como "una falta de atención a las necesidades de un niño(a), aun cuando se cuenta con los recursos para satisfacer dichas necesidades" (UNICEF, 2014, p. 4). En este estudio sólo se analizan las respuestas a la última pregunta sobre las posibles causas de negligencia hacia niños, y los comentarios adicionales que aportaran a la misma.

\section{Participantes}

Los participantes se obtuvieron mediante un muestreo no probabilístico por bola de nieve. Inicialmente, las investigadoras contactaron personalmente y por correo electrónico a personas conocidas que cumplían con los criterios de inclusión, invitándoles a participar en el estudio y a compartir el cuestionario con otros profesionales. Se incluyeron personas que tuvieran experiencia trabajando con niños en áreas de educación, psicología, seguridad, enfermería o trabajo social. La muestra se constituyó con 52 participantes, mayormente mujeres profesionales $(n=46)$ con edades entre 21 y $64(M=34.98$ años, $d s=$ 10.23) residentes de 23 municipios de Puerto Rico. La mayoría indicó tener grado de maestría (62\%), seguidas por $27 \%$ con grado de licenciatura y $11 \%$ con grado de doctorado. La muestra estuvo compuesta sobre todo por trabajadoras sociales (33\%) y maestras (33\%), seguidas por psicólogas (15\%) consejeras escolares (6\%), un abogado, una terapeuta ocupacional, una directora escolar, una gestora cultural y una gerente de proyectos educativos para jóvenes en riesgo de deserción escolar.

\section{Análisis de datos}

Los datos se analizaron siguiendo los pasos sugeridos por Braun y Clarke (2006); partiendo de la lectura y relectura de las respuestas se extrajeron 
todos los factores de riesgo, agrupándolos posteriormente bajo temas más amplios. Se excluyó del análisis un solo caso que enfocó sus respuestas en el maltrato por abuso sexual en lugar de la negligencia infantil. El resto de las respuestas se incluyeron en el análisis independiente al estilo o extensión presentado por el participante, ya que el propósito era identificar todos los posibles factores de riesgo.

Se creó una matriz en la que cada línea representaba un participante y se generaron columnas en la medida que se extraían factores de riesgo por caso. Una vez identificados y codificados, se procedió a revisarlos de nuevo para agruparlos por temas en una nueva matriz. Este proceso fue guiado por los intereses teóricos de la investigación, lo que Braun y Clarke (2006) describen como análisis temático teórico, y resultó en diez temas que serán descritos en el siguiente apartado. Se documentó la frecuencia con la que se mencionaron cada uno de los temas, considerándolo un indicador de la importancia o peso que le atribuyen los participantes. Posteriormente, se diseñó un esquema con los temas organizados por niveles de proximidad al niño, determinados según las cuatro estructuras interconectadas que constituyen el ambiente de desarrollo: el microsistema, el mesosistema, el exosistema y el macrosistema (Bronfrenbrenner, 1979).

\section{Resultados}

Del análisis de las respuestas de los profesionales, emergieron diez temas o variables de riesgo: la falta de destrezas parentales; la falta de interés y responsabilidad; pobreza de tiempo, uso y abuso de sustancias controladas; problemas de salud mental; embarazo a temprana edad; bajo nivel socioeconómico; falta de apoyo social y familiar; maltrato intergeneracional; y exclusión institucional. A continuación, se describen cada una y, en los casos que fue posible, se acompañan por citas directas de las personas entrevistadas.

\section{Falta de destrezas parentales}

Una de las principales causas de la negligencia, según los profesionales, es la falta de destrezas parentales. Con esto se refieren a la falta de información, apoyo, paciencia y motivación que tienen los padres para cumplir con sus responsabilidades y para lidiar con las diferentes etapas de desarrollo de sus hijos. Se menciona también la falta de inteligencia emocional y de destrezas de comunicación con sus hijos. Los padres no saben cómo manejar a sus hijos y no cuentan con las herramientas para guiarlos hacia una adolescencia saludable. Además, indican que los adultos desconocen las implicaciones legales de la negligencia.

[...] en ocasiones cuando el menor cursa el nivel superior, los padres se desligan de su obligación y en esta edad el menor está pasando por muchos cambios, retos y presiones de grupo. La ausencia y la falta de supervisión de sus padres puede influir de forma negativa en la toma de decisiones [...] 
No es que no los dejen respirar, pero tampoco es que estén completamente ausentes en sus vidas. Maestra, 35 años de edad.

\section{Falta de interés y responsabilidad}

En términos generales, los profesionales hablan de una distorsión en las prioridades de los cuidadores y una renuncia a las responsabilidades de crianza. Señalan que el trabajo, la obtención de bienes materiales y las relaciones o los problemas de pareja pasan a ser más importantes que el bienestar de los niños. Mencionan también que los padres se distraen con el uso de aparatos tecnológicos y redes sociales. Hay casos en los que simplemente se releva o se evade por completo la responsabilidad de cuidado.

La diferencia entre un padre responsable en la crianza de su hijo y un padre que no lo es, determina la manera que van a realizar sus deberes escolares y el esfuerzo con que realizan su trabajo diario. El estudiante que tiene padres responsables siempre quiere estar al día, aunque se haya ausentado. Maestra, 53 años de edad.

Las parejas se unen en ocasiones sin tener claras sus responsabilidades y tratan de delegar (la responsabilidad de cuidar al menor) a cualquiera sin medir las consecuencias. Trabajadora social, 64 años de edad.

\section{Pobreza de tiempo}

Padres con demasiado trabajo, sobrecarga, estilos de vida ajetreados y falta de organización y tiempo. Los profesionales entienden que las jornadas de trabajo exhaustivas privan a los padres del tiempo necesario para el cuidado de niños. Aún más cuando los padres necesitan más de un empleo para poder vivir. Si bien el trabajo les permite a los padres cumplir con algunas necesidades materiales del menor, relegan otras que consideran menos importantes.

[...] el manejo del tiempo, para el adulto trabajador, en una sociedad con motor económico capitalista, favorece la satisfacción de lo inmediato antes que el cumplimiento de lo importante. Maestro, 33 años de edad.

[...] hoy día se vive con tanta prisa que olvidamos muchos detalles en la vida de los menores [...] por ejemplo: la madre soltera con menores, quizás en su ajetreo de trabajar para llevar el sustento al hogar pierde esa sensibilidad de sentarse a estudiar con el menor o algo tan simple como llevarlo a una cita médica. Trabajadora social, 28 años de edad.

\section{Uso y abuso de sustancias controladas}

El alcoholismo y el uso de drogas son comunes en padres negligentes. Las profesionales indican que los cuidadores negligentes suelen ser dependientes de alguna sustancia psicoactiva. 
[...] puede darse el caso donde el factor alcoholismo afecta al hogar y a la familia [...] todo es una cadena y, lamentablemente, quien más se afecta son los menores. Trabajadora social, 28 años de edad.

\section{Problemas de salud mental}

Trastornos como la depresión, ansiedad y estrés son reconocidos como factores de riesgo por los profesionales. Además de la mención específica de estos diagnósticos, los profesionales hacen referencia a las condiciones de vida, que podrían provocarles dichos síntomas. Mencionan, por ejemplo, la sobrecarga laboral, la falta de servicios, la falta de apoyo tanto familiar como de agencias gubernamentales, que no ofrecen orientación o ayuda cuando las familias lo solicitan. Entienden que cuando esas variables interactúan generan las condiciones idóneas para que surjan eventos de negligencia.

\section{Embarazo a temprana edad}

Los profesionales indican que algunas personas tienen hijos aun cuando no están capacitados para entender y atender las necesidades de otro; lo describen como jóvenes o "niños criando niños".

Muchas personas se hacen padres a temprana edad sin tener los recursos o las herramientas necesarias tanto físicas, mentales y económicas para asumir una responsabilidad [...] como lo es tener a su cargo otra persona. Directora escolar, 52 años de edad.

\section{Bajo nivel socioeconómico}

Los profesionales hicieron referencia a la falta de dinero, el bajo ingreso o falta de recursos económicos, la baja o nula educación formal, pobreza y marginación. La situación económica en la que se encuentren los padres afecta también al menor, limitando el acceso que puedan tener a ciertos servicios. El nivel educativo también podría afectar las expectativas educativas que tienen los padres de sus hijos, de ahí que no se involucren en sus obligaciones escolares. Algunas respuestas dan cuenta de una situación de pobreza como la descrita por Lewis (1971). Por ejemplo, se menciona que hay padres que prefieren gastar su dinero en "necesidades momentáneas y pasajeras", como en su apariencia física y lujos que les permitan presumir de una capacidad adquisitiva que realmente no tienen.

Los padres están tratando de satisfacer otras necesidades que consideran prioritarias [...]. Gestora cultural, 29 años de edad.

Tienen hijos por las ayudas gubernamentales, pues así reciben más dinero. Maestra, 38 años de edad. 


\section{Falta de apoyo}

Se menciona la falta de apoyo en el caso de padres o madres solteras que no tienen familiares cercanos y la ausencia de una de las dos figuras paternas, debido a problemas con la ley.

La falta de una de las figuras paternas por problemas con la ley afecta mayormente a los grupos más desventajados económicamente. Maestro, 33 años de edad.

\section{Maltrato intergeneracional}

Las familias en las que ocurren eventos de negligencia suelen tener un historial de abuso físico, emocional o sexual. Los profesionales hacen referencia a "ciclos de maltrato familiar" y "patrones de maltrato". Tomando en cuenta que las escasas estadísticas oficiales de violencia suelen subestimar las incidencias de negligencia, en 1996 y 2013 se reportaron cifras similares de casos de maltrato, superando los 34,200 referidos (Maclay-Muñiz, Nieves, \& Rivera-Lugo, 2017). Aunque se desconocen los cambios que pueden haber surgido entre esos años, se puede inferir que la situación de violencia intrafamiliar se ha mantenido relativamente estable con el pasar de los años. A pesar de que este dato no nos refiere al maltrato intergeneracional, sí apunta a un contexto social violento que lo nutre.

\section{Exclusión institucional}

La falta de acceso a servicios básicos -y de calidad-, el alto costo de vida, la desigualdad de género, desigualdad económica, pobreza y falta de apego a derechos humanos, fueron algunas de las razones que se mencionaron como detonantes de la negligencia. Señalan que el cierre de escuelas y los servicios médicos inadecuados limitan las oportunidades educativas e incrementan los problemas de las familias, especialmente las que viven en pobreza. Estas injusticias y desigualdades crean familias desvinculadas de sus deberes parentales. Existe también una ausencia de orientación y educación que ayude a los padres a entender las diferentes necesidades de un menor en cada etapa de desarrollo.

El seguimiento del profesional escolar es clave en la prevención y [eliminación] de la negligencia. Las familias necesitan apoyo para cumplir con sus responsabilidades. Maestra, 50 años de edad.

La negligencia es un problema complejo. El mismo no sólo se debe a situaciones familiares, sino que se vincula a las condiciones de vida de la sociedad puertorriqueña. En Puerto Rico existe mucha desigualdad económica, los servicios sociales son escasos [...] Nuestra personalidad está continuamente disminuida por un régimen colonial [...] que controla los aspectos económicos y políticos de la Isla. Todas estas situaciones crean frustración, confusión y coraje que se manifiesta en el desinterés en cambiar, en aceptar el status quo. Considero que las familias negligentes 
han perdido la esperanza y proyectan este hecho en la falta de atención y compromiso con el óptimo desarrollo de sus hijos e hijas. Trabajadora social, 63 años de edad.

Como se mencionó anteriormente, los factores de riesgo se organizaron por niveles de proximidad al niño. En la Figura 2 se propone un esquema socioecológico de factores de riesgo para la negligencia infantil y se incluye la frecuencia con la que los profesionales mencionaron cada uno de los factores.

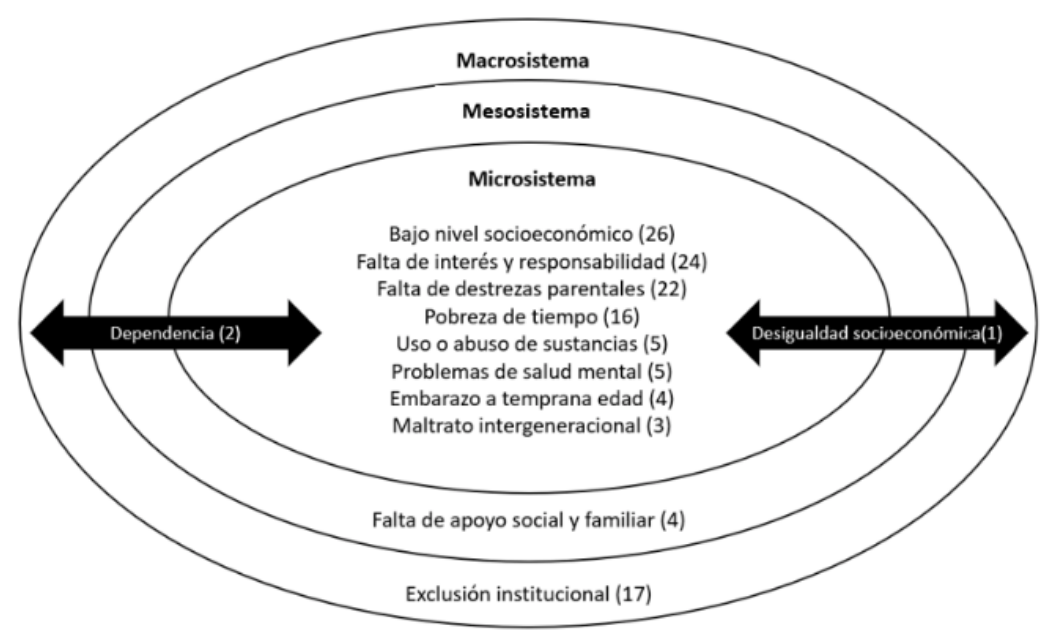

Figura 2. Esquema ecológico de factores de riesgo para la negligencia infantil.

Figura 2. Esquema ecológico de factores de riesgo para la negligencia infantil.

Además de los factores de riesgo que conforman los tres sistemas socioecológicos, el esquema propuesto contiene dos vectores o procesos que atraviesan los tres sistemas. Para Lewin (1952), los individuos participan de una serie de espacios de vida, como el hogar, la escuela o el trabajo, que son concebidos bajo la influencia de varios vectores de fuerza. Estos influyen sobre el individuo y determinan la dirección en la que se mueve en su entorno (Lewin, 1935).

Los dos vectores que forman parte del esquema son la dependencia tanto económica como política, y la desigualdad socioeconómica. La dependencia, en este caso producto del colonialismo, se manifiesta de varias formas en los diferentes niveles socioecológicos: en ayudas económicas institucionales, en instituciones, y la dependencia alimentaria del país. Todas éstas influyen sobre el funcionamiento psicológico de los individuos (Fanon, 1961; Hook, 2005). La desigualdad socioeconómica se mencionó como un obstáculo al acceso de servicios sociales, oportunidades y derechos. Esta, inevitablemente afecta y determina el curso de vida de las familias en riesgo.

Ambos vectores se contemplaron como fuerzas que influyen sobre el funcionamiento familiar y determinan sus dinámicas internas y externas. En palabras de un participante, son condiciones que "crean frustración, confusión y coraje" y "familias desvinculadas de sus deberes y responsabilidades para con sus criaturas y la sociedad en general. Trabajadora social, 63 años de edad. 


\section{Discusión}

Este estudio tenía por objetivo identificar factores de riesgo para la negligencia hacia niños en Puerto Rico desde la perspectiva profesional, empleando un análisis ecológico. Las respuestas ofrecidas por los profesionales fueron de utilidad para elaborar un esquema ecológico. Estas, aunadas a la evidencia empírica existente y el análisis de los modelos conceptuales, permiten inferir que la negligencia es un fenómeno multicausal, enriqueciendo así la conceptualización del fenómeno bajo estudio. Se pudo evidenciar que la negligencia es producto de carencias en diferentes subsistemas de interacción social. En congruencia, los resultados de este estudio sugieren que los abordajes teóricos psicológicos, sociológicos e interaccionales muestran limitaciones tanto conceptuales como metodológicas para explicar el fenómeno de la negligencia desde sus perspectivas unidisciplinarias.

Los profesionales entienden que hay características individuales de las madres y los padres, y factores familiares como la falta de apoyo, y sociales como la falta de servicios esenciales, que exponen a los niños a un mayor riesgo de negligencia. La variable que se mencionó con mayor frecuencia fue el bajo nivel socioeconómico, seguido por la falta de destrezas parentales, la falta de interés y responsabilidad por cuidar bien de sus hijos(as). Otros factores de riesgo importantes fueron la falta de tiempo para cuidar de sus hijos, y la falta de educación formal. De estos, el nivel socioeconómico (Duva \& Metzger, 2010; Eckenrode et al., 2014), la falta de destrezas parentales y la falta de educación formal (Counts, Buffington, Chang-Rios, Rasmussen, \& Preacher, 2010; Wolock \& Horowitz, 1984) son variables cuya relación con la negligencia ha sido documentada. Sin embargo, la falta de interés, responsabilidad y tiempo para cuidar adecuadamente de los hijos(as) no son temáticas tratadas en la literatura sobre negligencia. Estas causantes podrían estar asociadas a un sistema económico en el que es más urgente atender el trabajo y otras necesidades materiales antes que las necesidades de los niños(as).

Los factores de riesgo enumerados y descritos por los profesionales podrían organizarse bajo, al menos, tres de los sistemas propuestos: microsistema, mesosistema y macrosistema.

\section{Microsistema}

En ese sistema se incluyen características como la situación económica familiar, la falta de educación formal y la falta de interés y responsabilidad por parte de los cuidadores, el estrés parental, y el uso o abuso de sustancias controladas. Estas variables evidencian dificultades económicas, y en la literatura especializada son asociadas con la negligencia (Barnhart \& Maguire-Jack, 2016; Child Welfare Information Gateway, 2012; Duva \& Metzger, 2010; Slack et al., 2011).

Los problemas de salud mental y física, y el uso de sustancias pueden limitar la capacidad de los cuidadores para atender adecuadamente de 
sus hijos (Slack et al., 2011), ya sea porque se encuentran en un estado de incapacidad o porque no se cuenta con recursos para el cuidado del menor (Hornor, 2014). Por otra parte, el nivel socioeconómico de la familia presenta retos importantes para el cuidado de niños. Las condiciones económicas afectan el acceso a servicios médicos, la nutrición y la calidad de la vivienda, entre otros servicios vinculados con la salud. En este estudio, algunas variables relevantes para la manifestación de la negligencia hacia los niños sugieren interacciones interesantes que han sido también documentadas en la literatura especializada. Por ejemplo, el exceso de horas de trabajo y la sobrecarga de responsabilidades académicas y económicas podrían ser detonantes de estrés en los cuidadores; el estrés a su vez puede incidir en la forma en que estos interactúan con sus hijos (Bazon, Martínez Avila de Mello, Bérgamo, \& Faleiros, 2010; Slack et al., 2011).

En comparación con los otros sistemas considerados, se recabó un mayor número de factores de riesgo pertenecientes al microsistema. Revisiones sistemáticas recientes sobre factores de riesgo para la negligencia han concluido que los predictores más importantes de la negligencia han sido características parentales particulares como historial de ofensas criminales, historial de problemas de salud mental o psiquiátricos, y bajo nivel educativo (Mulder, Kuiper, Van der Put, Stams, \& Assnik, 2018). En el caso del presente estudio, esto podría deberse a que se mantiene un enfoque convencional sobre la conceptualización de la negligencia, que presta un mayor énfasis al rol del cuidador como perpetrador (Zuravin, 1999 en Dubowitz, Pitts, \& Black, 2004). Si bien son relevantes las variables mencionadas por los profesionales, deben analizarse en conjunto con los sistemas bajo los cuales se producen las características particulares del microsistema.

\section{Mesosistema}

En este nivel se detectó una variable: la falta de apoyo social y familiar. El apoyo social percibido por los cuidadores y trabajadores sociales ha sido vinculado previamente al maltrato infantil (Rajendran, Smith, \& Videka, 2015).

En términos de interacciones entre sistemas, Dixon, Browne y Hamilton-Giachritsis (2009) encontraron que el apoyo social y la estabilidad financiera pueden considerarse factores protectores contra la transmisión intergeneracional del maltrato infantil. Contrario a estos resultados, Bartlett, Raskin, Kotake, Nearing y Easterbrooks (2014) encontraron que el apoyo que percibe la madre de su familia y su comunidad no reduce la probabilidad de negligencia infantil por parte de sus madres.

En este nivel también se mencionó la ausencia de uno de los cuidadores por problemas con la ley. Ambos nos remiten al concepto de empobrecimiento social que discuten Garbarino y Sherman (1980), y que suele potencializar el maltrato hacia niños. Las familias consideradas en mayor riesgo de maltrato tienen una mayor necesidad de acceso a redes 
de apoyo y perciben sus comunidades como lugares inadecuados para la crianza.

\section{Macrosistema}

La toma de decisiones políticas que deriva en problemas como el cierre de escuelas y la falta de servicios sociales de calidad, se clasificó como exclusión institucional en este estudio, y formaría parte del macrosistema. Este tipo de políticas son incongruentes con los derechos de la niñez y ubican a las agencias gubernamentales en una posición que podría alentar la negligencia, al desatender también las necesidades médicas, educativas, recreativas, entre otras, de los niños. Además, crean ambientes comunitarios inapropiados para los niños.

Así mismo, las probabilidades de negligencia infantil aumentan cuando condiciones como las que se han descrito en otros niveles - pobreza, la falta de recursos vecinales y el aislamiento social - se extienden al contexto social y comunitario (Korbin \& Krugman, 2014, p. 34). Por el contrario, las comunidades ricas en capital social, con actividades centradas en la familia, centros de cuidado y un buen sistema de transporte público pueden crear un ambiente propicio para que las familias protejan a sus niños (Korbin \& Krugman, 2014, p. 35).

\section{Limitaciones}

La limitación principal de este estudio fue la ausencia de preguntas de seguimiento que permitieran enriquecer la descripción de los factores de riesgo mencionados por los profesionales. Por la misma razón, el alcance de las respuestas no es apto para hacer inferencias sobre la negligencia en diferentes etapas de la niñez y por condición de género. Esto es importante, ya que se ha documentado que el riesgo de ser víctimas de negligencia suele disminuir a medida que el menor crece (Korbin \& Krugman, 2014; Sledjeski et al., 2009), y que algunos tipos de negligencia como la educativa, son más comunes en varones (Disdier et al., 2015). Contrario a esa evidencia, una de las participantes indicó que, a medida que los jóvenes van creciendo, aumenta la falta de supervisión, pese a que los jóvenes están pasando por experiencias que ameritan el seguimiento de los adultos. En cuanto al género, ninguna de las respuestas apuntó a distinciones entre niños y niñas. Estudios posteriores podrían ayudar a distinguir entre grupos de edad y delimitar posibles relaciones entre las variables mencionadas por los profesionales.

En segundo lugar, las participantes fueron predominantemente mujeres del área metropolitana de Puerto Rico. Esta situación presenta un riesgo de sesgo en la información obtenida. El uso de un muestreo probabilístico podría ofrecer una perspectiva profesional más extensa y precisa de los factores de riesgo para la negligencia infantil.

Por último, aunque existe literatura internacional sobre la caracterización de la negligencia, es poca o nula la que se refiere a Puerto 
Rico en particular. Por lo tanto, se imposibilita la comparación y discusión de los hallazgos con otros estudios que analicen la perspectiva de una población similar.

\section{Conclusión}

Es importante recalcar que las opiniones profesionales no necesariamente están exentas de los prejuicios y mitos que permean a la sociedad puertorriqueña, sobre todo en relación con las comunidades empobrecidas (Harris \& Robinson, 2019; Morris et al., 2018). Se ha planteado que la intención de reportar el manejo y el desenlace de los casos de maltrato identificados se ve afectada por la clase social, raza y etnicidad de los denunciados (Jonson-Reid, Drake, \& Kohl, 2009; Lu et al., 2004; Tufford, Bogo, \& Asakura, 2015). Esto podría conducir, por ejemplo, a una cierta tolerancia o normalización de comportamientos negligentes que no se consideran lo suficientemente graves, o llevar a un mayor énfasis en el comportamiento de familias de bajo nivel socioeconómico.

Es decir, algunos comportamientos podrían juzgarse con mayor severidad cuando la población bajo observación es una de bajo nivel socioeconómico, en comparación a cuando son de un nivel más acomodado. De la misma forma, ciertas carencias materiales, psicológicas o educativas de los cuidadores podrían denominarse incorrectamente como negligencia. Un mayor número de preguntas y una indagación más profunda sobre las respuestas ofrecidas ayudarían a clarificar distinciones como las antes mencionadas.

Los resultados recopilados en este estudio se suman a la limitada literatura disponible sobre las causas de la negligencia hacia niños en Puerto Rico, entendiéndola como un problema relacionado pero distinto al abuso infantil. Ofrece también información que puede ser de utilidad para la redacción de propuestas de políticas públicas y la elaboración de planes de prevención e intervención para reducir o eliminar la negligencia. La voz y la experiencia de quienes interactúan diariamente con familias y niños es esencial tanto para la prevención como para la reducción de dicho fenómeno.

El modelo ecológico se propone como un marco conceptual idóneo para obtener una perspectiva más amplia del fenómeno de la negligencia; identificar variables críticas y sus interacciones. Esto permite identificar de forma más precisa las condiciones necesarias y suficientes para que ocurran conductas negligentes hacia niños en el contexto puertorriqueño. En la medida en que crezca ese cuerpo de conocimiento, se podrán construir alternativas para la transformación de cada uno de los subsistemas ecológicos que influyen en el desarrollo y bienestar de los niños y niñas. Es esa, finalmente, la tarea progresista: "estimular y posibilitar, en las más diversas circunstancias, la capacidad de intervención en el mundo, jamás lo contrario.” (Freire, 2012, p. 72). 


\section{Referencias}

Associated Press. (5 de mayo del 2017). Educación presenta lista de cierre de escuelas. Metro Puerto Rico. Recuperado de https://www.metro.pr/pr/no ticias/2017/05/05/educacion-presenta-lista-cierre-escuelas.html

Ávila-Claudio, R. (29 de agosto del 2017). Reportan 2,883 querellas contra hogares sustitutos de menores. Metro Puerto Rico. Recuperado de https://www.metro.pr/pr/noticias/2017/08/29/reportan-2883-quer ellas-hogares-sustitutos-menores.html

Barnhart, S., \& Maguire-Jack, K. (2016). Single mothers in their communities: The mediating role of parenting stress and depression between social cohesion, social control and child maltreatment. Children and Youth Services Review, 70, 37-45. https://doi.org/10.1016/j.childyouth.2016.09 .003

Bartlett, J. D., Raskin, M., Kotake, C., Nearing, K. D., \& Easterbrooks, M. A. (2014). An ecological analysis of infant neglect by adolescent mothers. Child Abuse \& Neglect, 38(4), 723-734. https://doi.org/10.1016/j.chiab u.2013.11.011

Baumrind, D. (1994). The social context of child maltreatment. Family Relations, 43(4), 360-368. https://doi.org/10.2307/585365

Bazon, M. R., Martínez Avila de Mello, I. L. M. A., Bérgamo, L. P. D., \& Faleiros, J. M. (2010). Negligência infantil: estudo comparativo do nível socioeconômico, estresse parental e apoio social. Temas em Psicologia, 18(1), 71-84.

Belsky, J. (1978). Three theoretical models of child abuse: A critical review. Child Abuse \& Neglect, 2(1), 37-49. https://doi.org/10.1016/0145-2134(78)9 $0005-4$

Belsky, J. (1980). Child maltreatment: An ecological integration. American Psychologist, 35(4), 320-335. https://doi.org/10.1037/0003-066X.35.4. 320

Belsky, J. (1993). Etiology of child maltreatment: A developmental- ecological analysis.Psychological Bulletin, 114(3), 413-434. https://doi.org/10.1037 /0033-2909.114.3.413

Berger, L. M., Font, S. A., Slack, K. S., \& Waldfogel, J. (2017). Income and child maltreatment in unmarried families: Evidence from the earned income tax credit. Review of Economics of the Household, 15(4), 1345-1372. https://d oi.org/10.1007/s11150-016-9346-9

Braun, V. \& Clarke, V. (2006) Using thematic analysis in psychology. Qualitative Research in Psychology, 3(2), 77-101. https://doi.org/10.1191 /1478088706qp063oa

Bronfenbrenner, U. (1979). La ecología del desarrollo humano: Experimentos en entornos naturales y diseñados. Paidós.

Bronfenbrenner, U., \& Mahoney, M. A. (1975). Influences on buman development. Holt McDougal.

Child Welfare Information Gateway. (2012). Child Abuse \& Neglect. Recuperado de https://www.childwelfare.gov/topics/can/

Children's Bureau. (2013). Child Welfare Outcomes 2010-2013. Report to Congress. U.S. Department of Health and Human Services, Children's Bureau. Recuperado de https://www.acf.hhs.gov/cb/resource/cwo-10-13 
Concepción Lizardi, A., \& Sánchez Peraza, L. R. (2017). Familias CAPACES: alternativas ecológicas para el referido ante el maltrato de menores en Puerto Rico. Revista Puertorriqueña de Psicología, 28(1),172-188.

Counts, J. M., Buffington, E. S., Chang-Rios, K., Rasmussen, H. N., \& Preacher, K. J. (2010). The development and validation of the protective factors survey: A self-report measure of protective factors against child maltreatment. Child Abuse \& Neglect, 34(10), 762-772. https://doi.org/ 10.1016/j.chiabu.2010.03.003

Departamento de la Familia. (2014). Plan nacional para la prevención del maltrato de menores en Puerto Rico 2014-2024. Recuperado de http://www.agencias.pr.gov/agencias/secretariado/Documents/Plan \%20nacional\%20para\%20la\%20prevenci\%C3\%B3n\%20del\%20maltrat o\%20de\%20menores\%20en\%20Puerto\%20Rico\%202014-2024.pdf

Disdier, O. M., Lugo, R., \& Irizarry, M. (2015). Perfil del maltrato de menores en Puerto Rico: año fiscal federal 2012-2013. Instituto de Estadísticas de Puerto Rico y Departamento de la Familia. Recuperado de http://www.e stadisticas.gobierno.pr

Dixon, L., Browne, K., \& Hamilton-Giachritsis, C. (2009). Patterns of risk and protective factors in the intergenerational cycle of maltreatment. Journal of Family Violence, 24(2), 111-122. https://doi.org/10.1007/s10896-008 $-9215-2$

Dubowitz, H. (2009). Tackling child neglect: A role for pediatricians. Pediatric Clinics of North America, 56(2), 363-378. https://doi.org/10.1016/j.pcl. 2009.01.003

Dubowitz, H., Black, M., Starr Jr, R. H., \& Zuravin, S. (1993). A conceptual definition of child neglect. Criminal Justice and Behavior, 20(1), 8-26. ht tps://doi.org/10.1177/0093854893020001003

Dubowitz, H., Pitts, S. C., \& Black, M. M. (2004). Measurement of three major subtypes of child neglect. Child Maltreatment, 9(4), 344-356 https://doi .org/10.1177/1077559504269191

Duva, J., \& Metzger, S. (2010). Addressing poverty as a major risk factor in child neglect: Promising policy and practice. Protecting Children, 25(1), 63-74.

Eckenrode, J., Smith, E. G., McCarthy, M. E., \& Dineen, M. (2014). Income inequality and child maltreatment in the United States. Pediatrics, 133(3), 454-461. https://doi.org/10.1542/peds.2013-1707

El Vocero. (22 de octubre de 2017). Senador Dalmau propone enmiendas para activar psicólogos en las escuelas. El Vocero. Recuperado de https://www.elvocero.com/gobierno/senador-dalmau-propone-enmi endas-para-activar-psic-logos-en-las/article_d9401360-b772-11e7-989bb7a7afbd $48 \mathrm{~d} 3 . \mathrm{html}$

Fanon, F. (1961). Los condenados de la Tierra. Fondo de Cultura Económica, México.

Fattah, E. A. (1989). The plight of crime victims in modern society. San Martín.

Freire, P. (2012). Pedagogía de la indignación: Cartas pedagógicas en un mundo revuelto. Siglo Veintiuno Editores S.A.

Friedrich, W. N., \& Boriskin, J. A. (1976). The role of the child in abuse: A review of the literature. American Journal of Orthopsychiatry, 46(4), 580-590. https://doi.org/10.1111/j.1939-0025.1976.tb00957.x 
Garbarino, J. (1977). The human ecology of child maltreatment: A conceptual model for research. Journal of Marriage and Family, 39(4), 721-735. http s://doi.org/10.2307/350477

Garbarino, J., \& Sherman, D. (1980). High-risk neighborhoods and high-risk families: The human ecology of child maltreatment. Child Development, 51(1), 188-198.

Gelles, R. J. (1973). Child abuse as psychopathology: A sociological critique and reformulation. American Journal of Orthopsychiatry, 43(4), 611-621. http s://doi.org/10.1111/j.1939-0025.1973.tb00830.x

Gil, D. G. (1969). Physical abuse of children: Findings and implications of a nationwide survey. Pedriatics, 44(5), 857-864.

Harris, A., \& Robinson, R. (2019). Child welfare workers perspective of poverty, and case substantiation (Tesis doctoral). California State University, Colorado.

Hook, D. (2005). A critical psychology of the postcolonial. Theory \& Psychology, 15(4), 475-503. https://doi.org/10.1177/0959354305054748

Hornor, G. (2014). Child neglect: Assessment and intervention. Journal of Pediatric Health Care, 28(2), 186-192. https://doi.org/10.1016/j.pedhc. 2013.10.002

Instituto de Estadísticas de Puerto Rico. (2016). Emigración neta se mantiene en su punto más alto en once años [Comunicado de prensa]. Instituto de Estadísticas de Puerto Rico, Estado Libre Asociado de Puerto Rico. Recuperado de https://www.noticel.com/ahora/20160915/la-emigracio n-neta-se-mantiene-en-su-punto-mas-alto-en-11-anos/

Ishida, K., Klevens, J., Rivera-García, B., \& Mirabal, B. (2013). Child maltreatment in Puerto Rico: Findings from the 2010 National Child Abuse and Neglect Data System. Puerto Rico Health Sciences Journal, 32(3), 124-131.

Jaschek, G., Carter-Pokras, O., He, X., Lee, S., \& Canino, G. (2016). Association of child maltreatment and depressive symptoms among Puerto Rican youth. Child Abuse \& Neglect, 58, 63-71. https://doi.org/10.1016/j.chia bu.2016.06.016

Jonson-Reid, M., Drake, B., \& Kohl, P. L. (2009). Is the overrepresentation of the poor in child welfare caseloads due to bias or need? Children and Youth Services Review, 31(3), 422-427. https://doi.org/10.1016/j.childyouth.20 08.09.009

Kilmer, R. P. \& Gil-Rivas, V. (2010). Responding to the needs of children and families after a disaster: Linkages between unmet needs and caregiver functioning. American Journal of Orthopsychiatry, 80(1), 135-142. https: //doi.org/10.1111/j.1939-0025.2010.01016.x

Kim, J. (2009). Type-specific intergenerational transmission of neglectful and physically abusive parenting behaviors among young parents. Children and Youth Services Review, 31(7), 761-767. https://doi.org/10.1016/j.ch ildyouth.2009.02.002

Korbin, J. E., \& Krugman, R. D. (2014). Handbook of child maltreatment. Springer. https://doi.org/10.1007/978-94-007-7208-3

Lewin, K. (1935). A dynamic theory of personality. McGraw-Hill.

Lewin, K. (1952). Field theory in social science. Harper \& Row. 
Lewis, H. B. (1971). Shame and guilt in neurosis. Psychoanalytic Review, 58(3), 419-438.

Ley para la Seguridad, Bienestar y Protección de Menores, Ley No 246 (2011). Recuperado de http://www.bvirtual.ogp.pr.gov/ogp/Bvirtual/leyesrefere ncia/PDF/Justicia/246-2011/246-2011.pdf

Lu, Y. E., Landsverk, J., Ellis-Macleod, E., Newton, R., Ganger, W., \& Johnson, I. (2004). Race, ethnicity, and case outcomes in child protective services. Children and Youth Services Review, 26(5), 447-461. https://doi.org/10. 1016/j.childyouth.2004.02.002

Lynch, M., \& Cicchetti, D. (1998). An ecological-transactional analysis of children and contexts: The longitudinal interplay among child maltreatment, community violence, and children's symptomatology. Development and Psychopathology, 10, 235-237. https://doi.org/10.1017 /S095457949800159X

Maclay-Muñiz, C., Nieves, I., \& Rivera-Lugo, C. (2017). El manejo de casos en maltrato a menores en Puerto Rico: èrelación eficiente y eficaz? Informes Psicológicos, 17(1), 13-34. http://dx.doi.org/10.18566/infpsic.v17n1a01

Martin, M. J., \& Walters, J. (1982). Familial correlates of selected types of child abuse and neglect. Journal of Marriage and the Family, 44(2), 267-276. h ttps://doi.org/10.2307/351537

Morris, K., Mason, W., Bywaters, P., Featherstone, B., Daniel, B., Brady, G., Bunting, L., Hooper, J., Mirza, N., Scourfield, J., \& Webb, C. (2018). Social work, poverty, and child welfare interventions. Child \& Family Social Work, 23(3), 364-372. https://doi.org/10.1111/cfs.12423

Mulder, T. M., Kuiper, K. C., van der Put, C. E., Stams, G. J. J., \& Assink, M. (2018). Risk factors for child neglect: A meta-analytic review. Child Abuse \& Neglect, 77, 198-210. https://doi.org/10.1016/j.chiabu.2018.01.006

National Research Council. (1993). Understanding child abuse and neglect. National Academy Press.

Oliveira, R. V., Maroco, J., \& Pais, L. G. (2012). The origin of maltreatment: An exploratory study on the intergenerational transmission of child abuse typologies. Interdisciplinaria, 29(2), 253-269.

Pardeck, J. T. (1989). Child abuse and neglect: Theory, research and practice. Gordon and Breach Science Publishers.

Patterson, G. (1982). Coercive family process. Castalia.

Platt, J. R. (1964). Science, strong inference-proper scientific method (the new baconians). Science, 146(3642), 347-353.

Proctor, L. J., \& Dubowitz, H. (2014). Child neglect: Challenges and controversies. En J. E. Korbin \& R. D. Krugman (Eds.), Handbook of child maltreatment (pp. 27-61). Springer.

Rajendran, K., Smith, B. D., \& Videka, L. (2015). Association of caregiver social support with the safety, permanency, and well-being of children in child welfare. Children and Youth Services Review, 48, 150-158. https://doi.org /10.1016/j.childyouth.2014.12.012

Sánchez Cesáreo, M., Sánchez Cardona, I. S., Beyer, M., González Jiménez, C. J., \& Bensinger, K. (2018). Factores asociados al abuso de menores: resultados de una intervención para el fortalecimiento de prácticas de crianza. Revista Puertorriqueña de Psicología, 29(1), 16-35. 
Sánchez Cesáreo, M., Santiago Rodríguez, B., Morales Boscio, A. M., Hernández Gierbolini, I., Toro Torres, J. I., \& Colón Jordán, H. (2016). Identificación y diseminación de intervenciones exitosas para la prevención del maltrato a menores en Puerto Rico. Revista Puertorriqueña de Psicología, 27(1), 10-24.

Sciarrino, N. A., Hernandez, T. E., \& Davidtz, J. (2018). Understanding child neglect: Biopsychosocial perspectives. Springer. https://doi.org/10.1007/97 8-3-319-74811-5

Slack, K. S., Berger, L. M., DuMont, K., Yang, M. Y., Kim, B., Ehrhard-Dietzel, S., \& Holl, J. L. (2011). Risk and protective factors for child neglect during early childhood: A cross-study comparison. Children and Youth Services Review, 33(8), 1354-1363. https://doi.org/10.1016/j.childyouth.2011.0 4.024

Sledjeski, E. M., Dierker, L. C., Bird, H. R., \& Canino, G. (2009). Predicting child maltreatment among Puerto Rican children from migrant and nonmigrant families. Child Abuse \& Neglect, 33(6), 382-392. https://doi.org /10.1016/j.chiabu.2008.11.004

Smith, J. A., \& Osborn, M. (2003). Interpretative phenomenological analysis. En J. A. Smith (Ed.), Qualitative psychology: A practical guide to methods (pp. 53-80). Sage.

Smith, J. D., Dishion, T. J., Shaw, D. S., Wilson, M. N., Winter, C. C., \& Patterson, G. R. (2014). Coercive family process and early-onset conduct problems from age 2 to school entry. Development and Psychopathology, 26(4pt1), 917-932. https://doi.org/10.1017/S0954579414000169

Smith, M. G., \& Fong, R. (2004). The children of neglect: When no one cares. Brunner-Routledge.

Spinetta, J. J., \& Rigler, D. (1972). The child-abusing parent: A psychological review. Psychological Bulletin, 77(4), 296-304. https://doi.org/10.1037/ h0032419

Thornberry, T. P., \& Henry, K. L. (2013). Intergenerational continuity in maltreatment. Journal of Abnormal Child Psychology, 41(4), 555-569. htt ps://doi.org/10.1007/s10802-012-9697-5

Torío-López, S., \& Peña-Calvo, J. V. (2006). Etiología y factores de riesgo de los malos tratos intrafamiliares a la infancia. Intervención desde la escuela. Revista Española de Pedagogía, 64(235), 525-544.

Tufford, L., Bogo, M., \& Asakura, K. (2015). How do social workers respond to potential child neglect? Social Work Education, 34(2), 229-243. https://d oi.org/10.1080/02615479.2014.958985

U.S. Department of Health \& Human Services, Administration for Children and Families, Administration on Children, Youth and Families (ACF), \& Children's Bureau. (2016). Child maltreatment 2014. Recuperado de htt ps://www.acf.hhs.gov/sites/default/files/cb/cm2014.pdf

UNICEF. (2014). Hidden in plain sight: A statistical analysis of violence against children. United Nations Children's Fund, UNICEF.

Vondra, J. I., \& Toth, S. L. (1989). Ecological perspectives on child maltreatment: Research and intervention. Early Child Development and Care, 42(1), 11-29. https://doi.org/10.1080/0300443890420102

Wiehe, V. R. (1989). Child abuse: An ecological perspective. Early Child Development and Care, 42(1), 141-149. 
Williams, S. E. (2017). Redrawing the line: An exploration of how lay people construct child neglect. Child Abuse \& Neglect, 68, 11-24. https://doi.or g/10.1016/j.chiabu.2017.03.014

Wolock, I., \& Horowitz, B. (1984). Child maltreatment as a social problem: The neglect of neglect. American Journal of Orthopsychiatry, 54(4), 530. https ://doi.org/10.1111/j.1939-0025.1984.tb01524.x 